Stem-cell ethics
$\begin{aligned} & \text { Korean bioethicists } \\ & \text { call for inquiry into } \\ & \text { cloning experiment } \\ & p 490\end{aligned}$

\title{
NASA devolves control of Cassini observations to research teams
}

Tony Reichhardt, Washington

Scientists gearing up for the most ambitious planetary mission in history say it will also be the most demanding for them. For the Cassini mission, NASA has shifted much of the hands-on planning from the agency's own centres to the instrument teams in the universities.

The US\$3.2-billion US-European spacecraft is set to arrive at Saturn on 1 July to begin a fouryear, 74-orbit tour of the giant gas planet, its rings and its moons. While a dozen instruments on the main orbiter study the Saturn system close up and map its electromagnetic field, the attached European-built Huygens probe will drop down in January to the surface of Titan, the planet's largest moon.

Similar in design to the Galileo

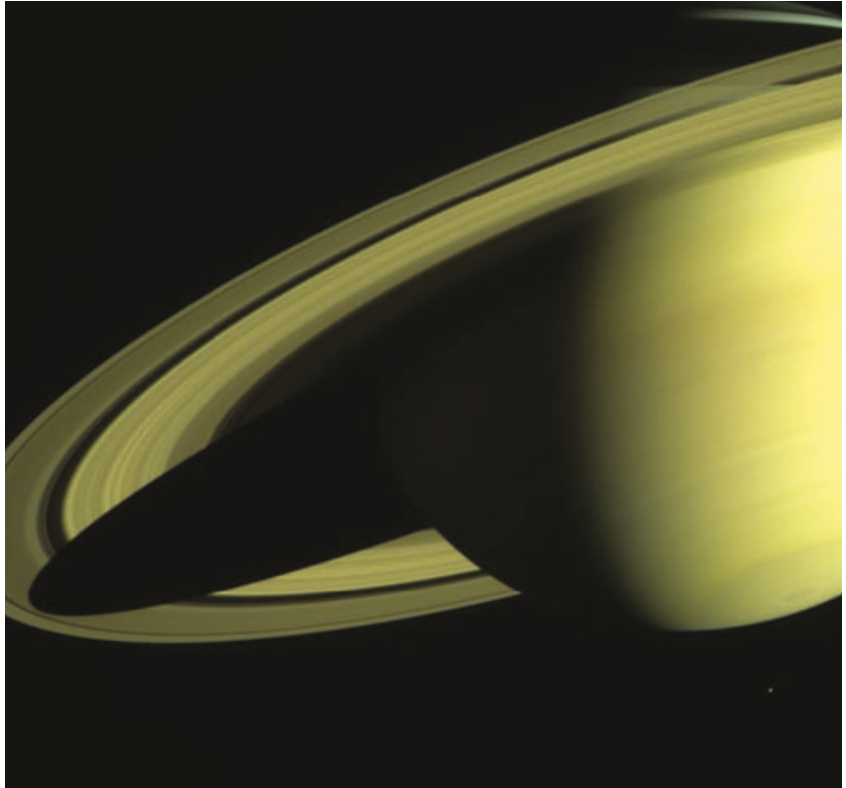

Getting closer: Saturn as seen from the Cassini spacecraft last month. experimental control that scientists gain by creating their own observing sequences. But, he says, the new strategy carries some risk. While all commands will be checked by JPL before they are sent to Cassini, to ensure that they do not jeopardize the spacecraft, some observations could be less efficient than they might be, as a dozen different teams are involved in the sequencing.

Scientists are reluctant to complain about funding, however, with Cassini's price tag reaching a cool $\$ 3.2$ billion. That includes $\$ 400$ million for the launch, $\$ 750$ million paid by European countries, and \$720 million to operate Cassini and keep the science teams funded until the end of the mission in 2008.

And Cassini's investigators are looking forward to the richest scientific bounty of their careers. mission that explored Jupiter and its moons in the 1990s, Cassini will be "like Galileo on steroids", says Torrence Johnson of the Jet Propulsion Laboratory in Pasadena, California, a member of the Cassini imaging team and former Galileo project scientist. Not only will Cassini cram far more observations into half the time, data from the spacecraft will pour in much faster than it did from Galileo, which was hampered by a jammed antenna.

Scientists have had far more responsibility for the details of the Cassini mission than they had with Galileo and with the Voyager missions that surveyed Jupiter, Saturn and the outer planets in the 1970s and 1980s and are now out in deep space. On those projects, investigators who had built cameras and other sensors requested where their instruments should be pointed and when, and technicians at JPL worked out the complex sequences of spacecraft manoeuvres needed to carry out the observations.

But when Cassini was being planned in the early 1990s, NASA decided that instrument teams would work the manoeuvres out for themselves - partly to give the scientists more control and partly to save money. A lot of the work was "thrown over the fence" to the outside scientists, says Stamatios Krimigis of the Johns Hopkins University Applied Physics Laboratory in Laurel, Maryland, principal investigator for one of Cassini's magnetospheric experiments.

That allowed JPL to cut its operational budget for Cassini by some $40 \%$ compared with that of Galileo, according to Mark Dahl, NASA's programme executive for Cassini. But project scientists complain that they have had to absorb the extra work without adequate funding from NASA.

The problem was compounded by another NASA money-saving decision back in 1992. Instead of mounting the cameras on a separate platform so that they could be directed independently of other instruments, they were fixed to the spacecraft body, making all Cassini's sensors interdependent. This makes it far more difficult to plan the observation manoeuvres. "The complexity of the job was underestimated," says Krimigis, a sentiment echoed by other Cassini investigators.

Larry Esposito of the University of Colorado in Boulder, who built Cassini's ultraviolet-imaging spectrograph, likes the additional
Close study of the intricate ring system should teach researchers about the dynamics of similar disks surrounding other stars. Saturn's moon Enceladus may turn out to be geologically active, with water geysers spouting above its icy surface. Titan, with its constant haze and Earth-like, nitrogen-rich atmosphere, is a mystery that planetary scientists have long wanted to crack. And because Cassini will return hundreds of thousands of images of the planet's swirling clouds, researchers will be able to create extensive movies of the turbulent atmosphere - something they were unable to do at Jupiter because of Galileo's antenna problem.

Cassini entered Saturn's sphere of gravitational influence in mid-May. The action heats up on 11 June, when the inbound spacecraft makes its first close pass of a moon, tiny Phoebe. Then on 1 July, Cassini's main rocket engine will fire for 94 minutes to brake the spacecraft into orbit around Saturn and begin four years of intensive exploration. Comparing the pace of those observations to the United States' best-known car race, Dahl says: "Scientists are looking forward to the start of the Indianapolis 500.” 